\title{
Supporting Information for Quantifying Nanoparticle Assembly States in Polymer Matrix Through Deep Learning
}

Eric Zhonghang Qu, ${ }^{1}$ Andrew Matthew Jimenez, ${ }^{2}$ Sanat K. Kumar, ${ }^{2}$ and Kai Zhang ${ }^{1, *}$

${ }^{1}$ Division of Natural and Applied Sciences, Duke Kunshan University, Kunshan, Jiangsu, 215300, China

${ }^{2}$ Department of Chemical Engineering, Columbia University, New York, New York, 10027, USA

\section{EFFECTS OF DATA AUGMENTATION}

Hue

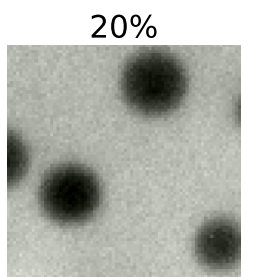

$50 \%$

Saturation

Brightness

Contrast

Distortion

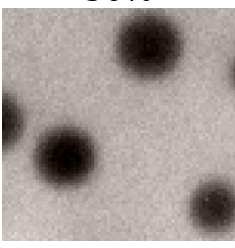

$50 \%$

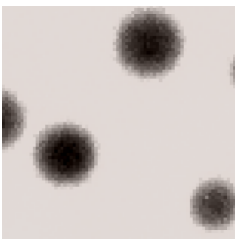

$50 \%$

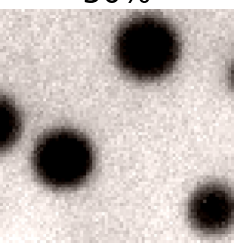

$10 \%$

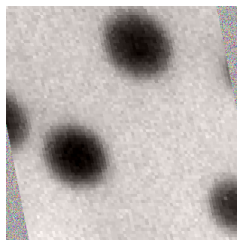

$10 \%$

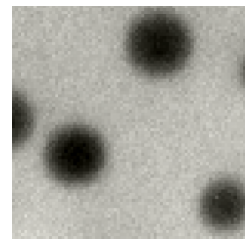

$25 \%$

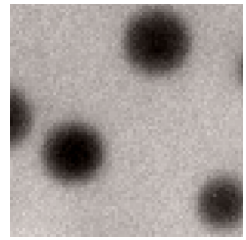

$25 \%$

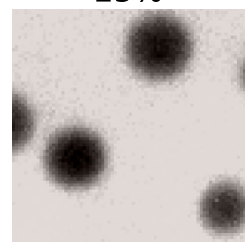

$25 \%$

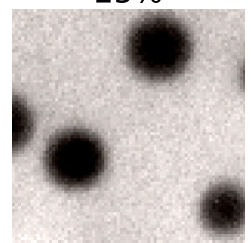

$5 \%$

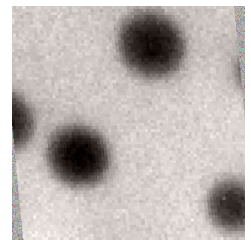

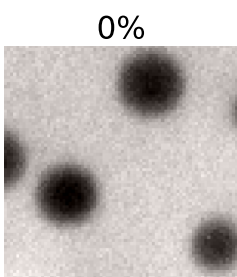

$0 \%$

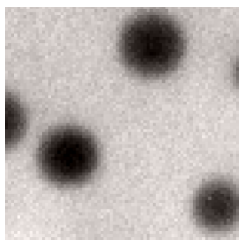

$0 \%$

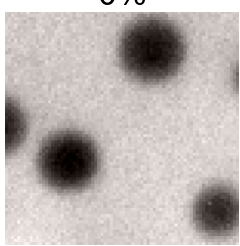

$0 \%$

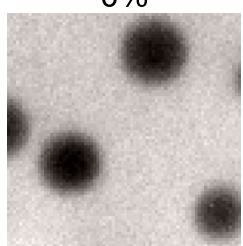

$0 \%$

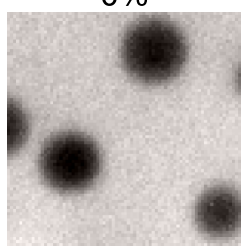

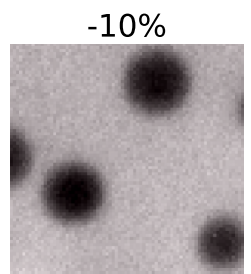

$-25 \%$

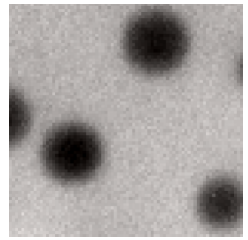

$-25 \%$

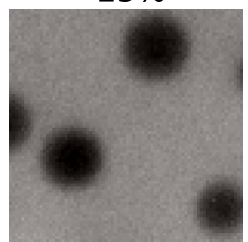

$-25 \%$

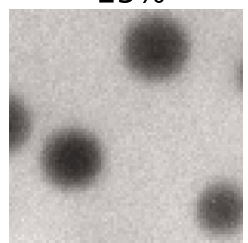

$-5 \%$

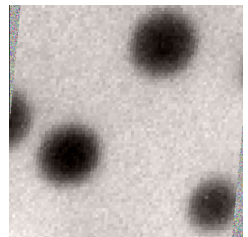

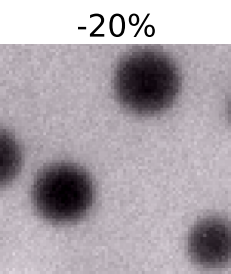

$-50 \%$

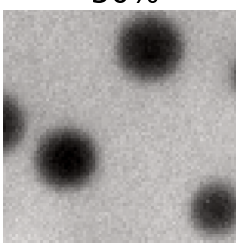

$-50 \%$

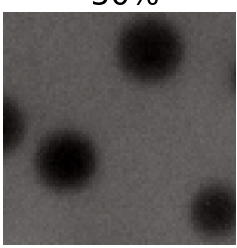

$-50 \%$

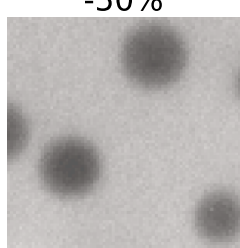

$-10 \%$

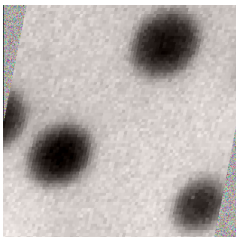

FIG. S1: Photometric and spatial distortions applied to an example image in DOPAD.

${ }^{*}$ Electronic address: kai.zhang@dukekunshan.edu.cn 


\section{EFFECTS OF TRANSFER LEARNING}

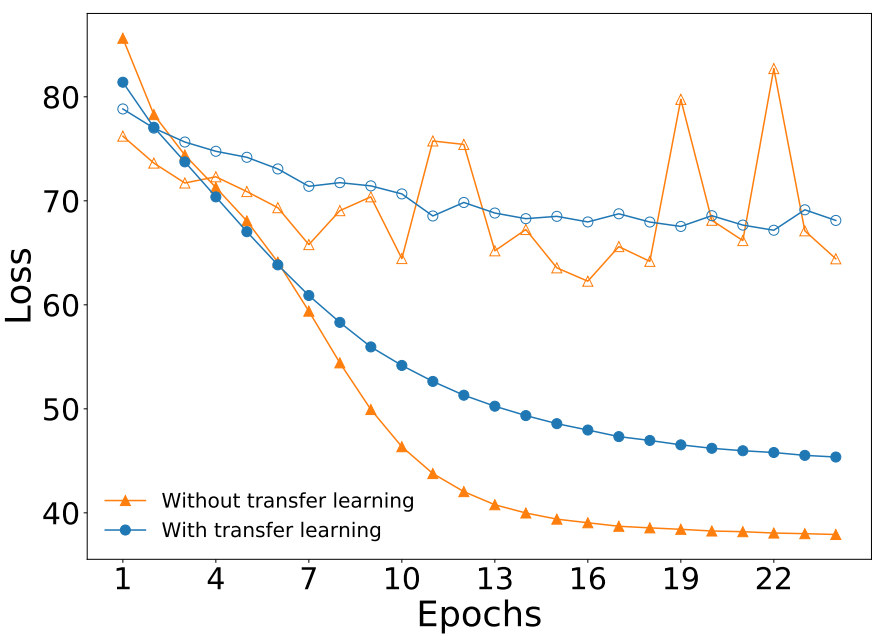

FIG. S2: Training (solid symbol) and validation (empty symbol) loss with and without transfer learning. 


\section{SLIDING WINDOW AND MERGING}

After the CNN model for DOPAD is optimized, a given large TEM image of arbitrary size can be analyzed by sliding a small square window over the entire image and then merging predictions from all windows. This idea is often used in problems in computer vision [1]. In our problem, if the original TEM image has $W \times H$ pixels, we apply the following workflow to extract locations of all NPs (Figure S3).

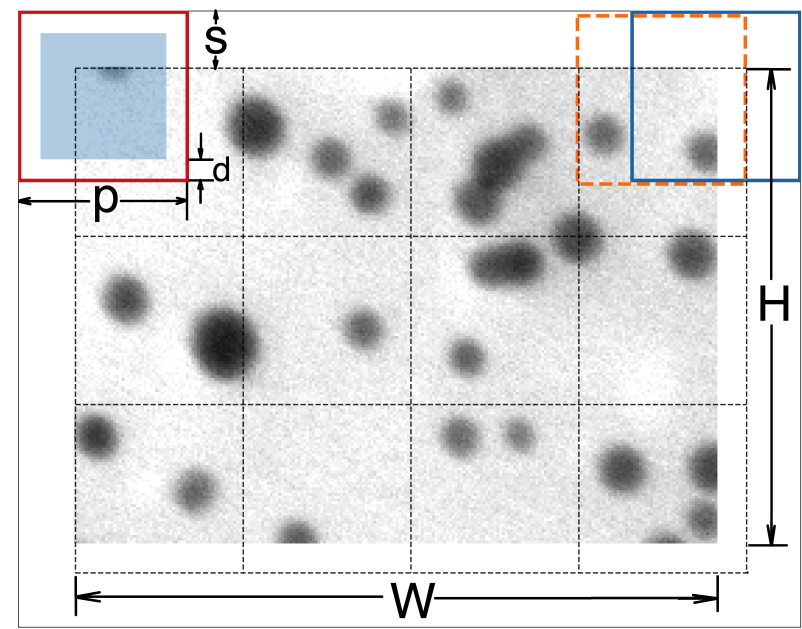

FIG. S3: Sliding-window method. The original large TEM image is of dimension $W \times H$. The method slides small square windows of length $p$ with stride $s$ to cover the entire image. Zero paddings are added around the original image to allow an integer number of cuts. In each sliding window, predicted bounding boxes entering margins of width $d$ are discarded. Only boxes within the shaded region are used.

- choose the size $p$ of the small square window with $p \times p$ pixels, such that the diameter of a NP in the image is about $1 / 10-1 / 4$ of the edge length of the square.

- choose the stride $s$ to slide the window. We set $s=\frac{p}{n_{s}}$ with a small integer, e.g. $n_{s}=2$ or 3 .

- choose the margin size $d$ for each window, e.g. $d=\frac{1}{16} p$. Any predicted bounding boxes whose vertex falls in the margin will be discarded. This helps to ignore truncated NPs at the edges of the window.

- add zero padding around the original TEM image to make it of size $W^{\prime} \times H^{\prime}=\left(W+W_{L}+W_{R}\right) \times\left(H+H_{U}+H_{D}\right)$ pixels. The added left $W_{L}$, right $W_{R}$, up $H_{U}$, down $H_{D}$ pixels should make sliding windows cover the whole image exactly. We set $W_{L}=s, W_{R}=p(\lfloor W / p\rfloor+1)-W+s, H_{U}=s, H_{D}=p(\lfloor H / p\rfloor+1)-H+s$.

- slide the $p \times p$ window over the padded image to take $n=n_{W} \times n_{H}$ small square views. With above choice of padding, $n_{W}=W^{\prime} / s-\left(n_{s}-1\right)$ and $n_{H}=H^{\prime} / s-\left(n_{s}-1\right)$.

- for each $p \times p$ square view obtained from the sliding-window method, rescale the image to $416 \times 416$ pixels and apply the CNN model to predict bounding boxes. This task can be paralleled in multicore processors since the temporal sequence to analyze different windows does not affect the final result. After discarding boxes that enter the margins of small squares, collect remaining boxes as final candinates into a set $\mathbb{C}$.

- apply non-max suppression algorithm to remove redundant boxes in $\mathbb{C}$ and obtain the set $\mathbb{B}$ of final predictions.

In set $\mathbb{C}$, it is expected that multiple bounding boxes enclosing the same NP are found from different sliding-windows. To remove redundant boxes such that only one box is predicted for each NP, the non-max suppression algorithm [2] is used. Once final bounding boxes are collected in set $\mathbb{B}$, the center of each NP can be readily extracted from box coordinates. The code then outputs coordinates $\left(x_{i}, y_{i}\right)$ of each located NP in a text file, together with an estimate of its diameter $\sigma_{i}=\min \left\{w_{i}, h_{i}\right\}$. If unsatisfactory, the machine-predicted results can be further improved by human researcher, either by manually adding missing particles into the output file or interactively modifying bounding boxes via online labeler [3]. Technical details about our deep-learning predictor are contained on the GitHub site https://dopad.github.io. 
4. FULL IMAGES FOR SAMPLES IN FIGURE 3, 4A, 4B AND 4C

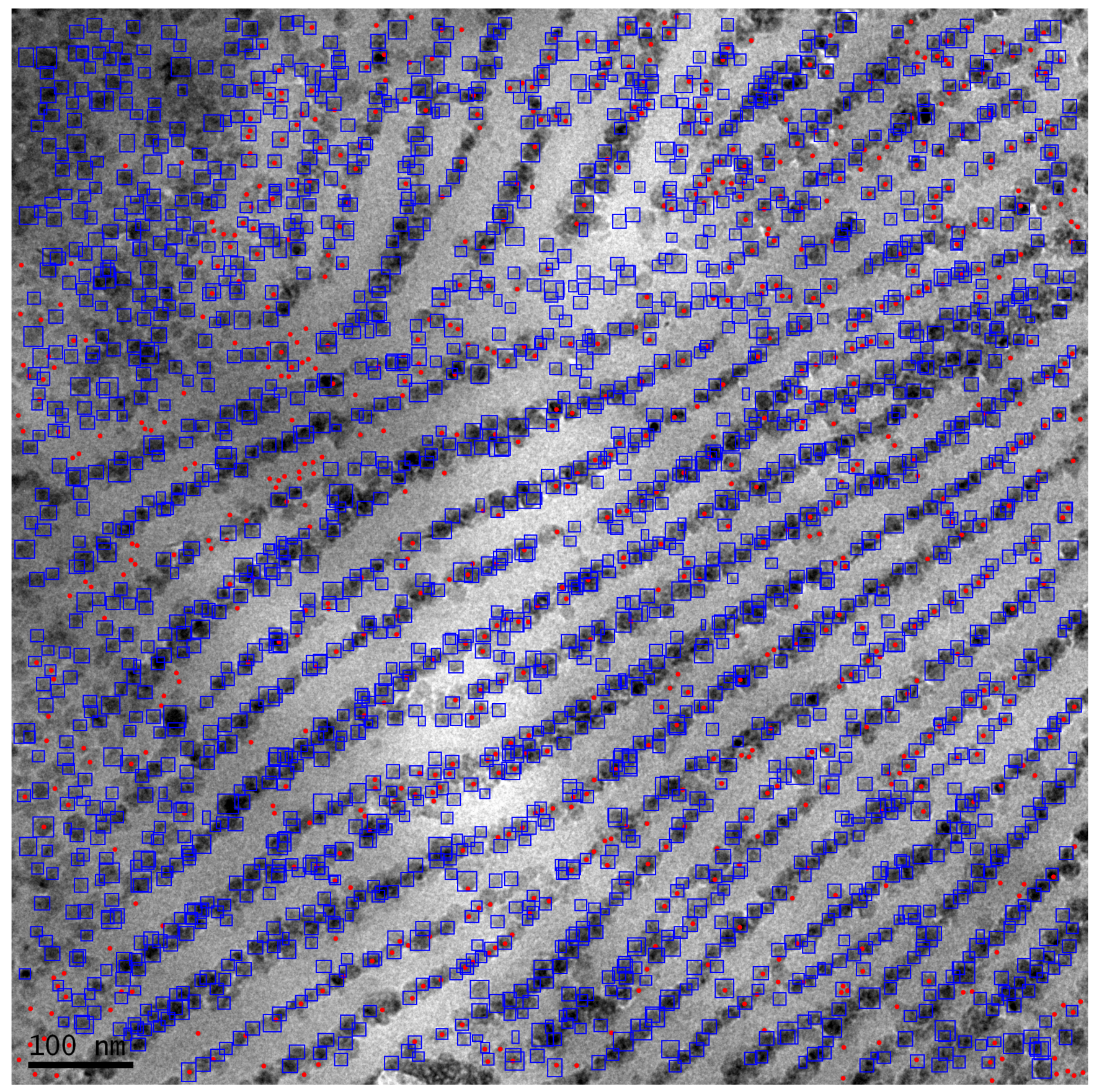

FIG. S4: Full image for the sample in Figure 4b with predicted bounding boxes (blue) and predicted locations by ImageJ (red dots). 


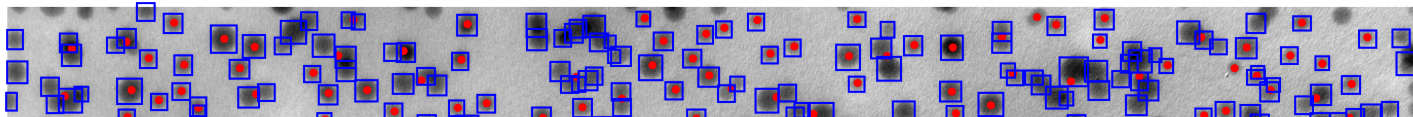

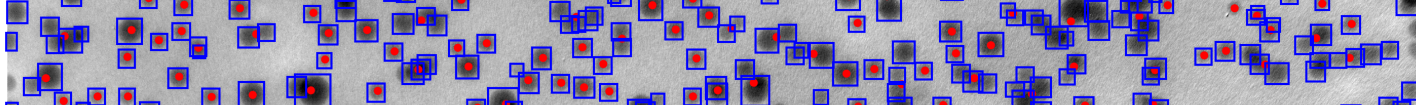

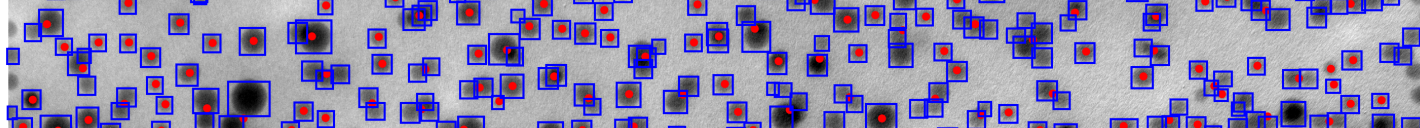

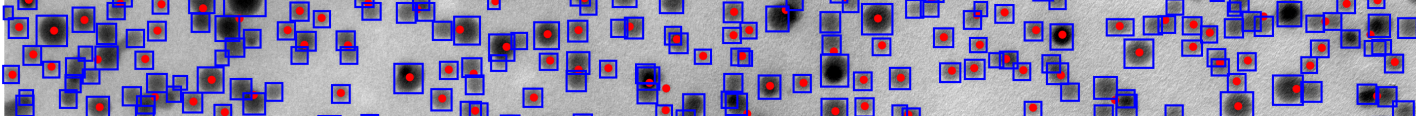

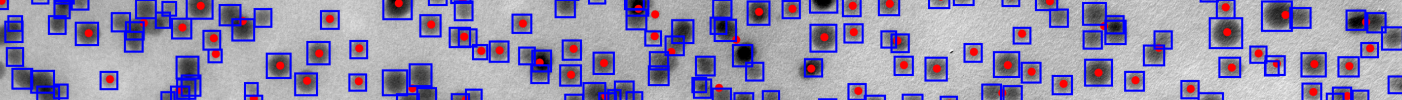

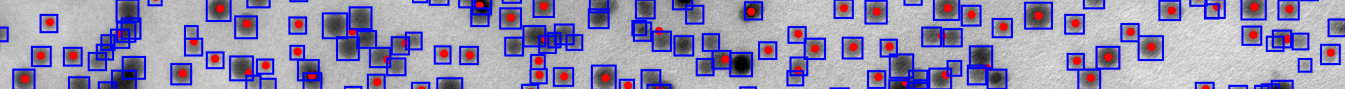

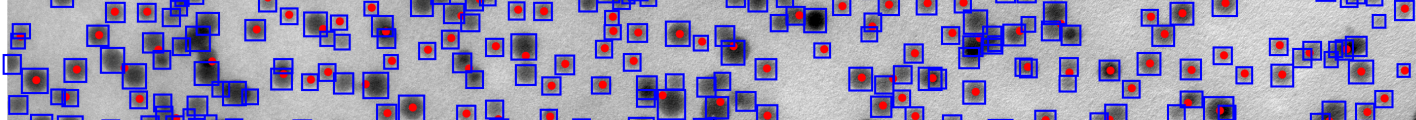

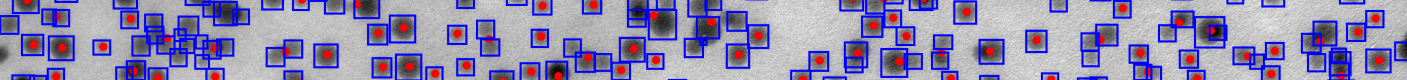

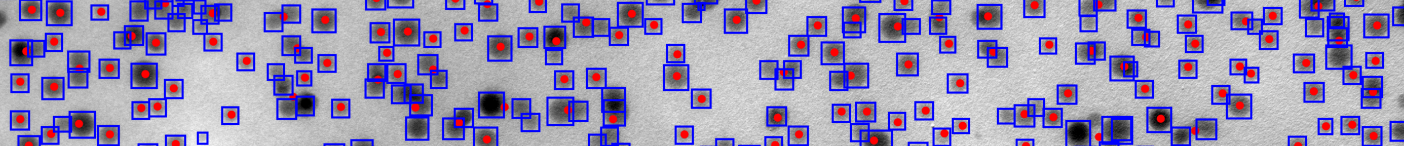

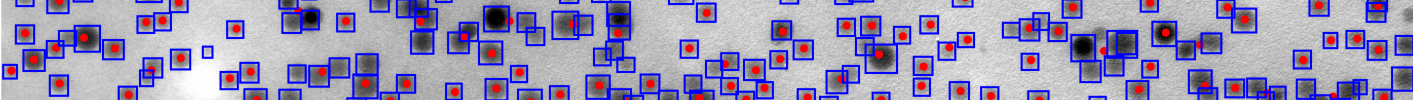

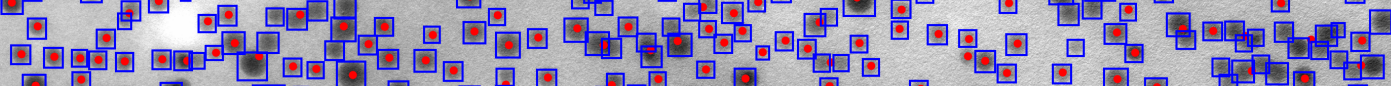

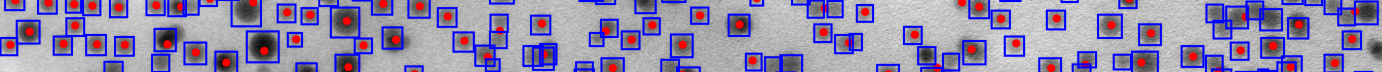

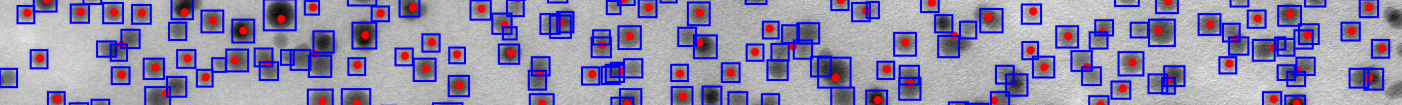

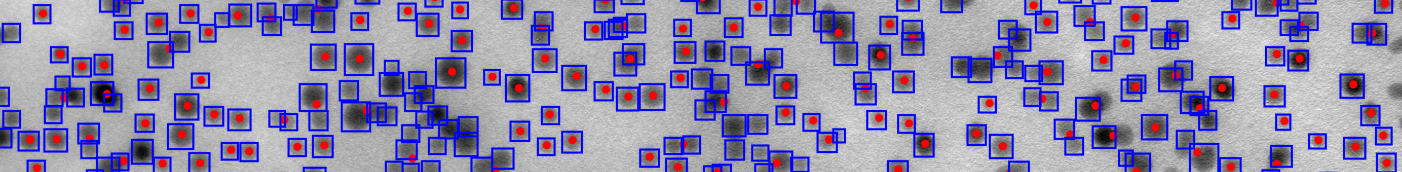

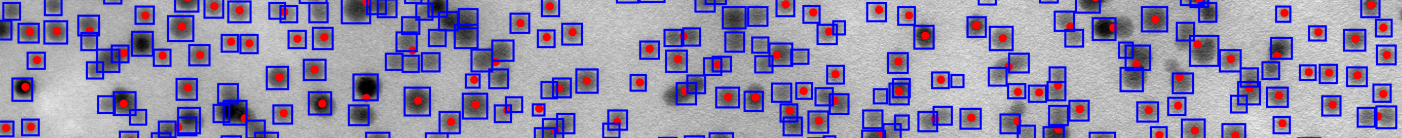

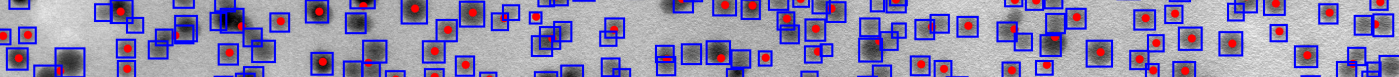

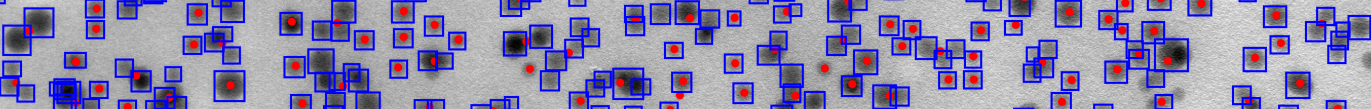

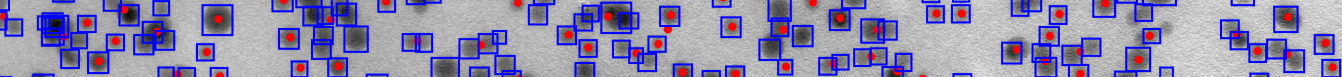

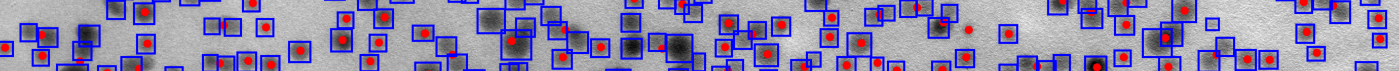

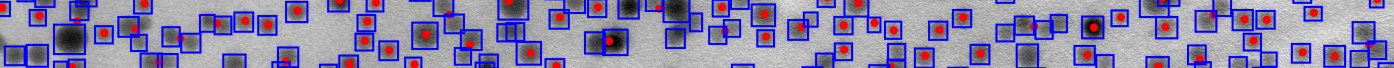

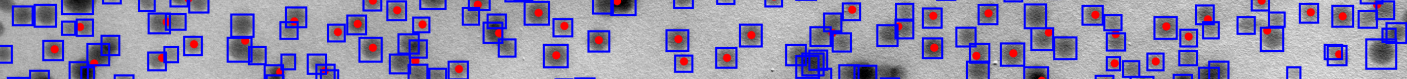

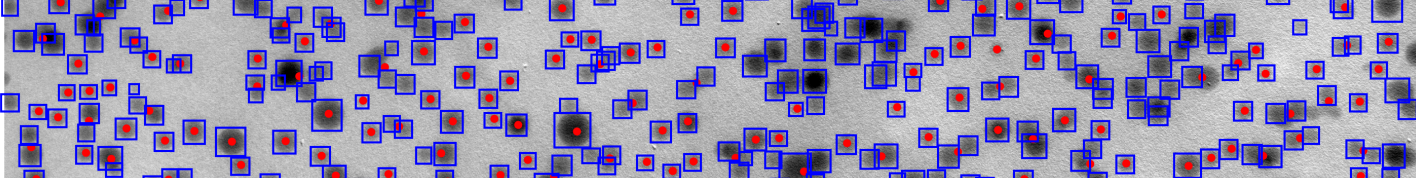

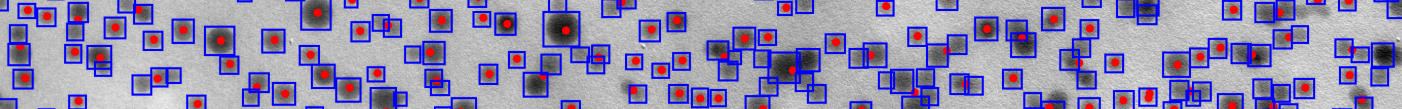
口

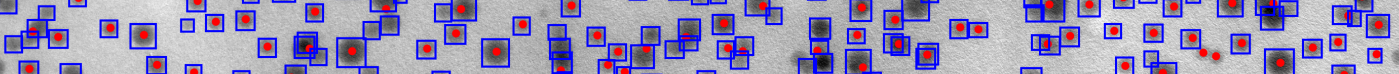

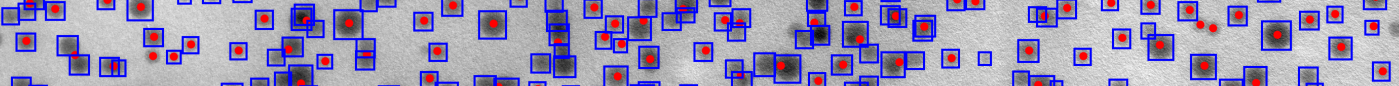

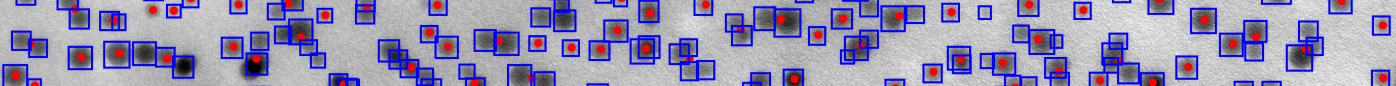

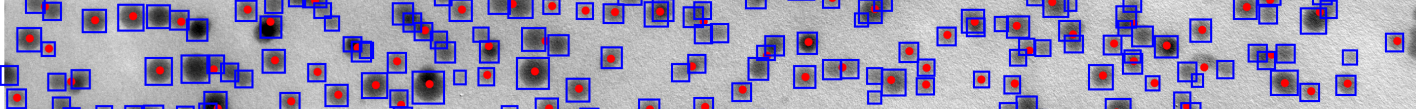

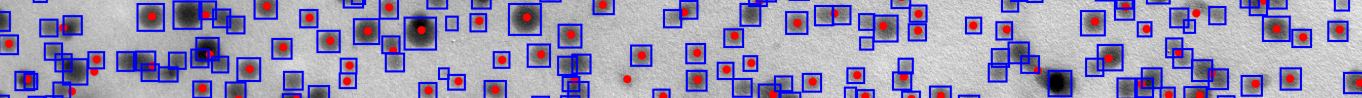

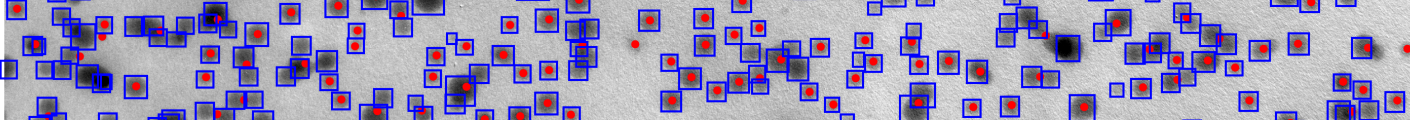

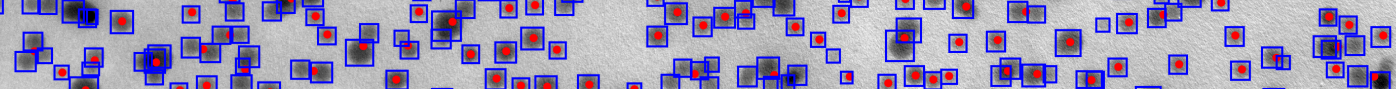

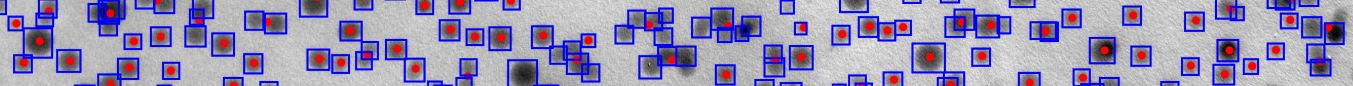

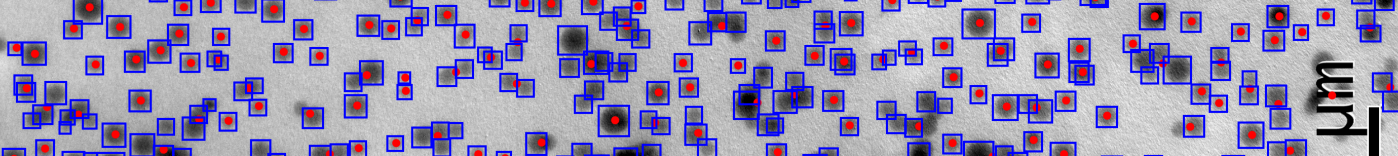

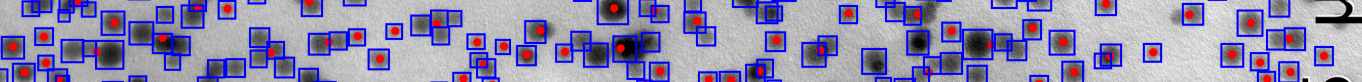

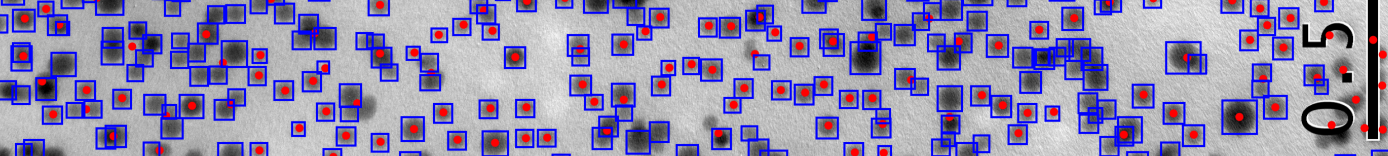
四

FIG. S5: Full image for the sample in Figure 3 with predicted bounding boxes (blue) and predicted locations by ImageJ (red dots). 
10 80.

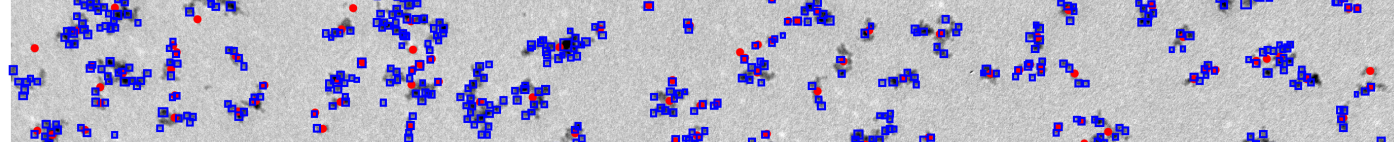
47. 4. - 7 of

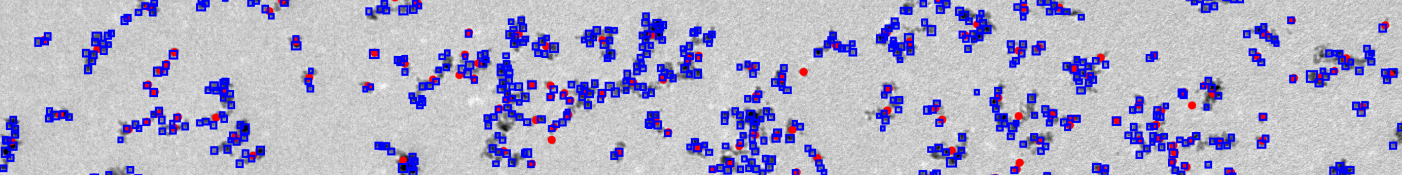

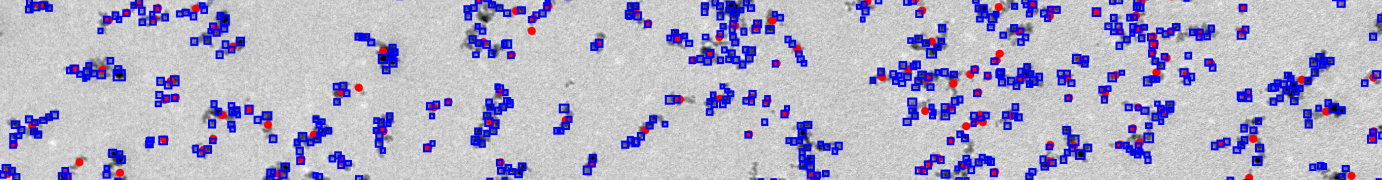

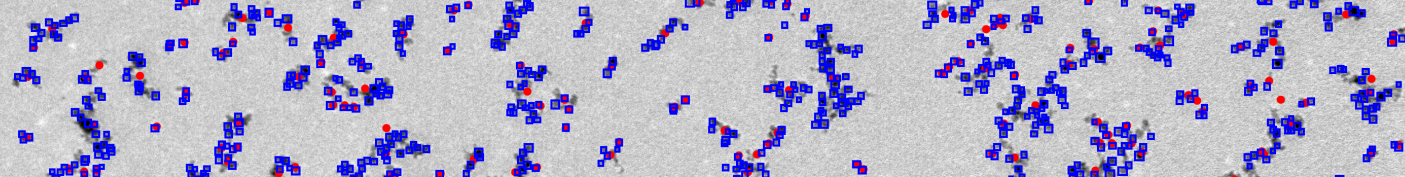

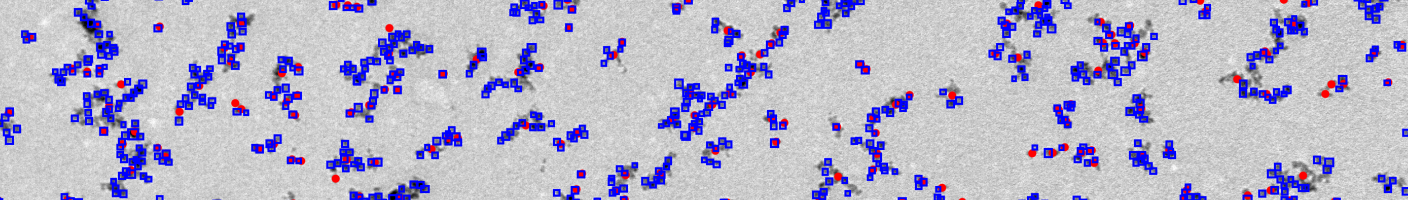

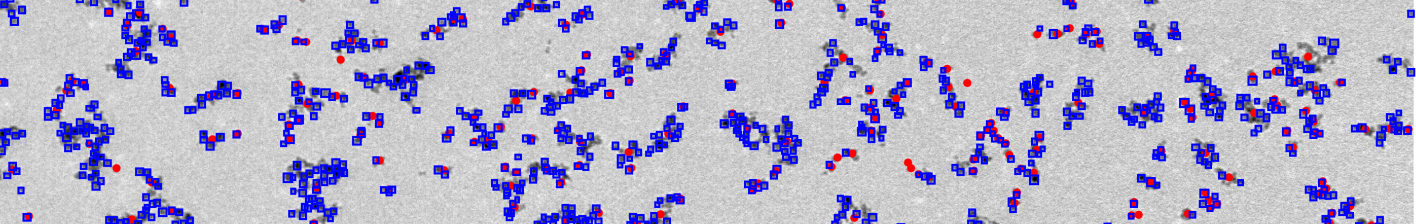
स. 5y 品2.

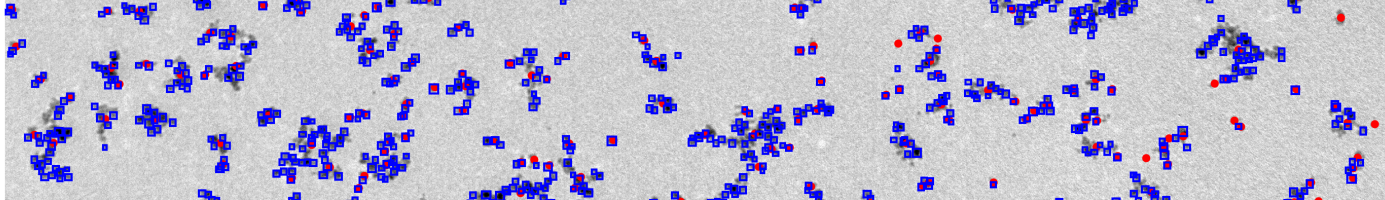

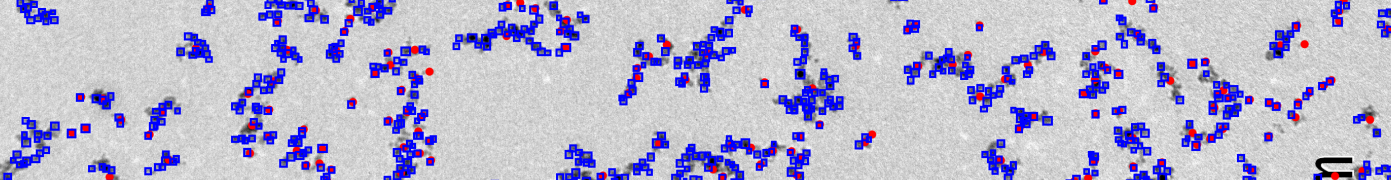
50

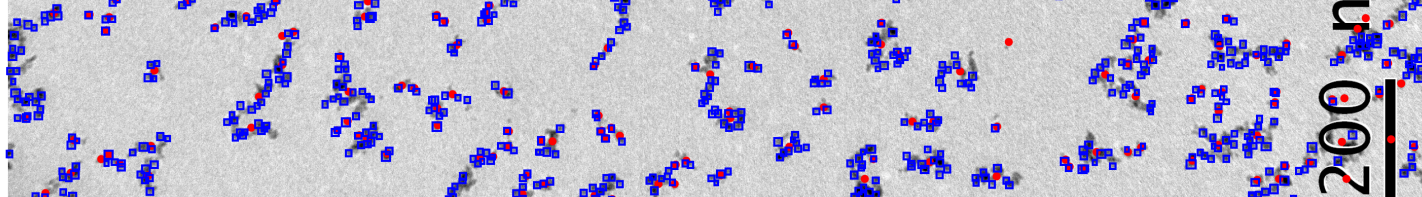
1. the 


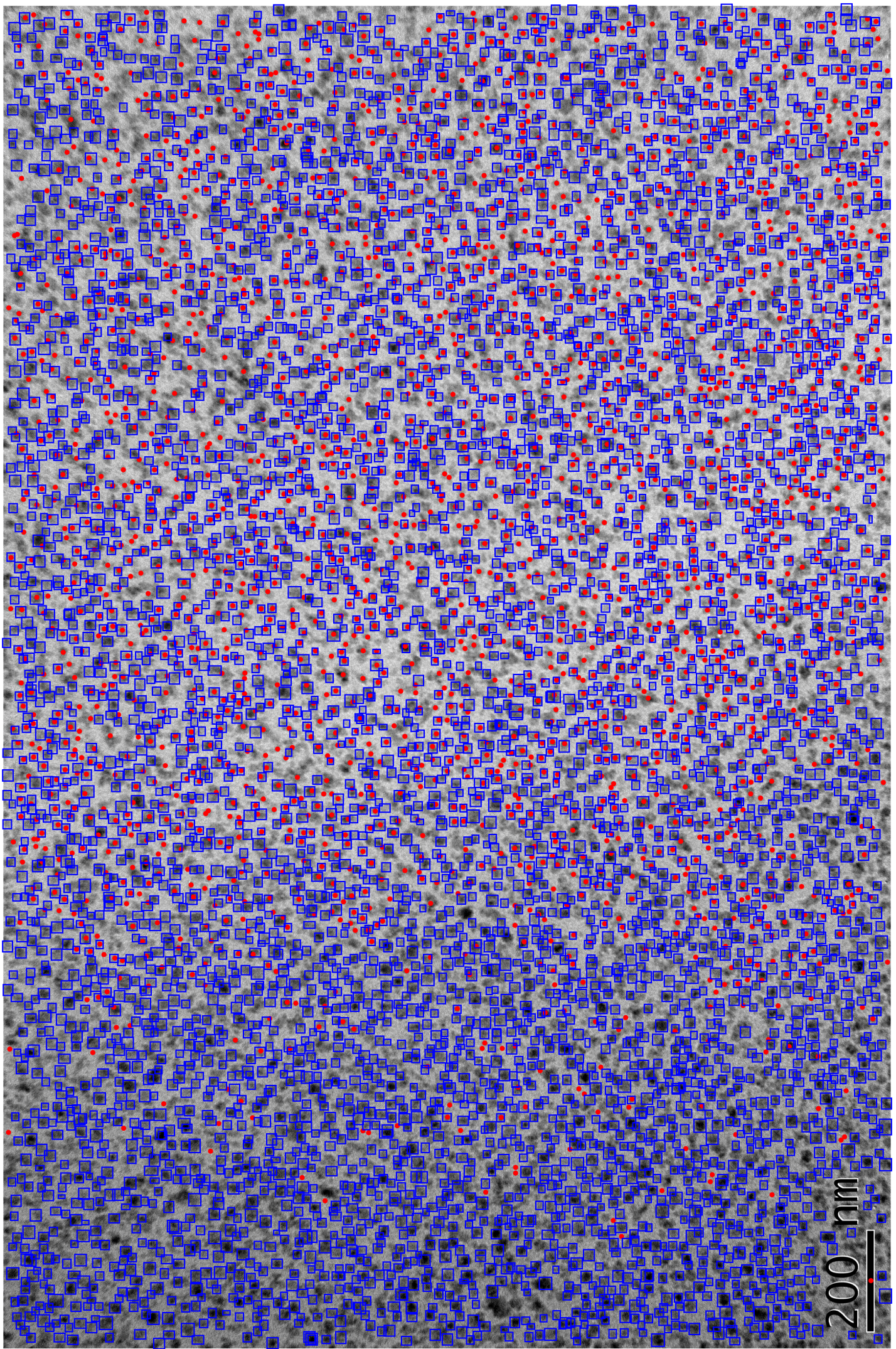

FIG. S7: Full image for the sample in Figure 4c with predicted bounding boxes (blue) and predicted locations by ImageJ (red dots). 
[1] A. Van Etten, arXiv preprint arXiv:1805.09512 (2018).

[2] A. Neubeck and L. Van Gool, in 18th International Conference on Pattern Recognition (ICPR'06) (IEEE, 2006), vol. 3, pp. 850-855.

[3] Colabeler, http://www.colabeler.com/. 